\title{
UNSTABLE SIMPLE MODES OF THE NONLINEAR STRING
}

\author{
$\mathrm{BY}$
}

THIERRY CAZENAVE (URA CNRS 189, Université Pierre et Marie Curie, Paris, France)

A.ND

FRED B. WEISSLER (URA CNRS 742, Université Paris XIII, Villetanneuse, France)

Abstract. We prove instability of high-energy simple modes for the nonlinear vibrating string equation

$$
\left\{\begin{array}{l}
u_{t t}-\left(a+b \int_{0}^{\pi} u_{x}^{2}(t, x) d x\right) u_{x x}=0 \\
u_{\mid \partial \Omega}=0
\end{array}\right.
$$

in $\Omega=(0, \pi)$, where $a \geq 0, b>0$.

Résumé. On montre l'instabilité des modes simples d'énergie assez grande pour l'équation des cordes vibrantes non-linéaire

$$
\left\{\begin{array}{l}
u_{t t}-\left(a+b \int_{0}^{\pi} u_{x}^{2}(t, x) d x\right) u_{x x}=0, \\
u_{\mid \partial \Omega}=0
\end{array}\right.
$$

dans $\Omega=(0, \pi)$, où $a \geq 0, b>0$.

1. Introduction. A classical model of the nonlinear vibrating string is given by the equation

$$
\left\{\begin{array}{l}
u_{t t}-\left(a+b \int_{0}^{\pi} u_{x}^{2}(t, x) d x\right) u_{x x}=0 \\
u_{\mid \partial \Omega}=0
\end{array}\right.
$$

in $\Omega=(0, \pi)$, where $a \geq 0, b>0$. This model has been extensively studied, for example, by Carrier [2], Bernstein [1], and Narashimha [8]. See also Dickey [4], Medeiros and Milla Miranda [7] and the references therein. Our main interest in this model is the stability of simple modes, i.e., solutions of the form $u(t, x)=\omega(t) \sin (j x)$, such solutions necessarily being periodic in time. Dickey [4] showed that small amplitude simple modes are stable. Here, we prove that simple modes of sufficiently large energy are unstable, i.e., that they have nontrivial unstable manifolds.

We study equation (1.1) using Fourier series. Set.ting

$$
u_{j}(t)=\sqrt{\frac{2}{\pi}} \int_{0}^{\pi} u(t, x) \sin (j x) d x,
$$

Received September 15, 1993.

1991 Mathematics Subject Classification. Primary 34D05, 35B40. 73K03, 35L70, 34B30, 33C45.

Key words and phrases. Nonlinear string equation, stable and unstable manifolds of periodic solutions. 
we obtain for the $u_{j}$ 's the following system of ODE's:

$$
u_{j}^{\prime \prime}+j^{2}\left(a+b \sum_{i=1}^{x} i^{2} u_{i}^{2}\right) u_{j}=0 .
$$

It follows that, given any integer $j \geq 1$, Eq. (1.1) has the particular (simple mode) solution $u(t, x)=\sqrt{\frac{2}{\pi}} \omega(t) \sin (j x)$ where $\omega$ solves the equation $\omega^{\prime \prime}+j^{2}\left(a+b j^{2} \omega^{2}\right) \omega=0$. To prove that simple modes are always unstable once they have sufficiently large energy, we consider a two-mode solution of (1.1) (or (1.2)), i.e., a solution of the system

$$
\left\{\begin{array}{l}
u_{j}^{\prime \prime}+j^{2}\left(a+b j^{2} u_{j}^{2}+b k^{2} u_{k}^{2}\right) u_{j}=0 \\
u_{k}^{\prime \prime}+k^{2}\left(a+b j^{2} u_{j}^{2}+b k^{2} u_{k}^{2}\right) u_{k}=0
\end{array}\right.
$$

where $j, k$ are integers such that $k>j \geq 1$. If $\left(u_{j}, u_{k}\right)$ solves system $(1.3)$, then $u(t, x)=$ $\sqrt{\frac{2}{\pi}}\left(u_{j}(t) \sin (j x)+u_{k}(t) \sin (k x)\right)$ solves Eq. (1.1). We set

$$
\gamma=\frac{k^{2}}{j^{2}}, \quad \sigma=j \sqrt{b}, \quad \nu=\frac{a}{b},
$$

so that (1.3) is equivalent to

$$
\left\{\begin{array}{l}
u^{\prime \prime}+\left(\nu+u^{2}+v^{2}\right) u=0 \\
v^{\prime \prime}+\gamma\left(\nu+u^{2}+v^{2}\right) v=0
\end{array}\right.
$$

where $j u_{j}(t)=u(\sigma t)$ and $k u_{k}(t)=v(\sigma t)$. The system (1.4) is a Hamiltonian system, whose conserved energy $E$, expressed in terms of $u, v, u^{\prime}, v^{\prime}$, is given by

$$
E\left(u, v, u^{\prime}, v^{\prime}\right)=\frac{u^{\prime 2}}{2}+\frac{v^{\prime 2}}{2 \gamma}+\nu \frac{u^{2}+v^{2}}{2}+\frac{\left(u^{2}+v^{2}\right)^{2}}{4}
$$

Our main result is the following.

ThEOREM 1.1. Assume that $\nu>0$ and let

$$
\gamma \in((m+1)(2 m+1),(m+1)(2 m+3)),
$$

for some nonnegative integer $m$. There exists $C>0$ such that if $E_{0} \geq C$, then there exists a two-dimensional submanifold $\mathcal{M}$ of the (three-dimensional) manifold $\left\{E\left(u, v, u^{\prime}, v^{\prime}\right)=\right.$ $\left.E_{0}\right\}$ with the following property. If $\left(u_{0}, v_{0}, u_{0}^{\prime}, v_{0}^{\prime}\right) \in \mathcal{M}$, and if $(u, v)$ is the solution of $(1.4)$ with initial data $\left(u_{0}, v_{0}, u_{0}^{\prime}, v_{0}^{\prime}\right)$, then $v$ and $v^{\prime}$ converge exponentially to 0 as $t \rightarrow \infty$, and there exists a solution $w$ of the equation $w^{\prime \prime}+\nu w+w^{3}=0$ with energy $\frac{w^{\prime 2}}{2}+\nu \frac{w^{2}}{2}+\frac{w^{4}}{4}=E_{0}$ such that $u-w$ and $u^{\prime}-w^{\prime}$ converge exponentially to 0 as $t \rightarrow \infty$.

Note that, given an integer $j \geq 1$, there exists $k>j$ such that $\gamma=k^{2} / j^{2}$ falls in one of the intervals defined in Theorem 1.1. Thus, since the system (1.3) is time reversible, Theorem 1.1 indeed proves that a simple mode $\sqrt{\frac{2}{\pi}} \omega(t) \sin (j x)$ of sufficiently large energy has indeed a nontrivial unstable manifold. 
In fact, more can be said. The first two intervals in Theorem 1.1 are $(1,3)$ and $(6,10)$. If we set $k=3 j, \forall j \geq 1$, then $\gamma=k^{2} / j^{2}=9$ independent of $j$. Theorem 1.1 thus implies the existence of an energy level above which all simple modes are unstable.

The reader may therefore wonder why, if $\gamma=9$ suffices to prove instability, the complete sequence of intervals in Theorem 1.1 was analyzed. To answer this question, we remark that, while the proof of Theorem 1.1 for just the interval $(1,3)$ is less technical than the full result, it does not suffice to show instability of all simple modes (consider $j=1)$. Once the second interval $(6,10)$ is included, however, the rest requires little additional work. Moreover, the sequence of intervals arises in a natural way using Jacobi polynomials.

Theorem 1.1 is proved by showing that a periodic solution of system (1.4) with sufficiently large energy and such that $v \equiv 0$ gives rise to a hyperbolic fixed point of the induced Poincaré map. The details of the proof follow the same outline as in our previous paper [3]; however, several new ingredients are needed to study the linearization of the Poincaré map. In particular, the limiting form (for high energy) of the linearized Poincaré map is defined using the system

$$
\left\{\begin{array}{l}
w^{\prime \prime}+w^{3}=0 \\
z^{\prime \prime}+\gamma w^{2} z=0
\end{array}\right.
$$

(See Sec. 3 below.) The second equation in (1.6) is an example of Hill's equation. In Chapter 2 of [6], the equation

$$
z^{\prime \prime}+(\gamma+Q) z=0
$$

where $\gamma$ is a parameter, is studied extensively. In particular, the real line is partitioned into a sequence of intervals, and the behavior of solutions to (1.7) depends on which interval contains $\gamma$. Even though $\gamma$ in (1.6) appears in a different part of the equation than in (1.7), our results concerning (1.6) are of the same type as in [6], except that we can also specify the intervals exactly.

The paper is organized as follows. In Sec. 2, we reduce the problem to the study of the linearized Poincaré map, which we do in Sec. 3 by considering the limiting system (1.6). Section 4 is devoted to several extensions of Theorem 1.1.

The authors are indebted to M. Balabane for some interesting remarks about polynomials, as well as to A. Haraux who suggested to us that the ideas in [3] could apply to the model (1.1).

2. Reduction to the linearized system. Throughout this section, $E_{0}>0$ is fixed. We define the mapping $T: \mathcal{U} \rightarrow \mathbf{R}^{2}$, where

$$
\mathcal{U}=\left\{(a, b) \in \mathbf{R}^{2} ; \frac{b^{2}}{2 \gamma}+\nu \frac{a^{2}}{2}+\frac{a^{4}}{4}<E_{0}\right\} .
$$

Given $(a, b) \in \mathcal{U}$, we consider the solution $(u, v)$ of (1.4) with initial data $\left(u, v, u^{\prime}, v^{\prime}\right)(0)=\left(0, a, u_{0}^{\prime}, b\right)$, where $u_{0}^{\prime}>0$ is defined by

$$
\frac{u_{0}^{\prime 2}}{2}+\frac{b^{2}}{2 \gamma}+\nu \frac{a^{2}}{2}+\frac{a^{4}}{4}=E_{0} .
$$


Since $u(0)=0$ and $u^{\prime}(0)>0$, it follows that $u(t)>0$ for $t>0$ and small. On the other hand, multiplying the equation for $u$ by $\sin (t / \sqrt{\nu})$ and integrating twice by parts on $(0, \pi / \sqrt{\nu})$, one sees easily that $u$ must have a zero on $(0, \pi / \sqrt{\nu})$. We denote by $\tau$ the first positive zero of $u$. Observe that $\tau$ itself depends on $(a, b)$. Finally, we define the mapping $T$ by

$$
T(a, b)=-\left(v(\tau), v^{\prime}(\tau)\right),
$$

for all $(a, b) \in \mathcal{U}$. It is clear that $T$ is of class $C^{1}$ and that

$$
T(0,0)=(0,0)
$$

We next define the linear operator $L \in \mathcal{L}\left(\mathbf{R}^{2}\right)$ as follows. Let $w_{0}^{\prime}>0$ be defined by

$$
\frac{w_{0}^{\prime 2}}{2}=E_{0}
$$

i.e., $w_{0}^{\prime}=\sqrt{2 E_{0}}$, and let $w$ be the solution of the equation

$$
w^{\prime \prime}+\nu w+w^{3}=0
$$

with initial data $w(0)=0$ and $w^{\prime}(0)=w_{0}^{\prime}$. Since $w(0)=0$ and $w^{\prime}(0)>0$, it follows that $w(t)>0$ for $t>0$ and small. On the other hand, by the same reasoning as above, $w$ must have a zero on $(0, \pi / \sqrt{\nu})$; we let $\rho$ denote the first positive zero of $w$. Given $(a, b) \in \mathbf{R}^{2}$, let $z$ be the solution of the (linear) equation

$$
z^{\prime \prime}+\gamma\left(\nu+w^{2}\right) z=0
$$

with initial data $z(0)=a$ and $z^{\prime}(0)=b$. We define $L \in \mathcal{L}\left(\mathbf{R}^{2}\right)$ by

$$
L(a, b)=-\left(z(\rho), z^{\prime}(\rho)\right)
$$

for all $(a, b) \in \mathbf{R}^{2}$. $T$ and $L$ are related as follows.

Proposition 2.1. DT $(0,0)=L$.

Proof. We know that $D T(0,0)$ exists. To prove it equals $L$, it suffices (since $T(0,0)=$ $(0,0))$ to show that

$$
\lim _{\Sigma l 0} \frac{T(\varepsilon(a, b))}{\varepsilon}=L(a, b),
$$

for all $(a, b) \in \mathbf{R}^{2}$. Fix $(a, b) \in \mathbf{R}^{2}$, and for $\varepsilon>0$ small enough, let $\left(u_{\varepsilon}, v_{\varepsilon}\right)$ be the solution of $(1.4)$ with initial data $\left(u_{\varepsilon}, v_{\varepsilon}, u_{\varepsilon}^{\prime}, v_{\varepsilon}^{\prime}\right)(0)=\left(0, \varepsilon a, c_{\varepsilon}, \varepsilon b\right)$, where $c_{\varepsilon}>0$ is defined by

$$
\frac{c_{\varepsilon}^{2}}{2}+\frac{\varepsilon^{2} b^{2}}{2 \gamma}+\nu \frac{\varepsilon^{2} a^{2}}{2}+\frac{\varepsilon^{4} a^{4}}{4}=E_{0},
$$

and let $\tau_{\varepsilon}$ be the first positive zero of $u_{\varepsilon}$ (see the definition of $T$ ). Set $z_{\varepsilon}(t)=v_{\varepsilon}(t) / \varepsilon$. Then

$$
\left\{\begin{array}{l}
u_{\varepsilon}^{\prime \prime}+\left(\nu+u_{\varepsilon}^{2}+\varepsilon^{2} z_{\xi}^{2}\right) u_{\varepsilon}=0, \\
z_{\varepsilon}^{\prime \prime}+\gamma\left(\nu+u_{\varepsilon}^{2}+\varepsilon^{2} z_{\varepsilon}^{2}\right) z_{\varepsilon}=0, \\
u_{\varepsilon}(0)=0, \quad u_{\varepsilon}^{\prime}(0)=c_{\varepsilon}, \quad z_{\S}(0)=a, \quad z_{\S}^{\prime}(0)=b .
\end{array}\right.
$$


It follows from $(2.5)$ that $\lim _{\varepsilon \downarrow 0} c_{\varepsilon}=\sqrt{2 E_{0}}$. Also,

$$
T(\varepsilon(a, b))=-\left(v_{\varepsilon}\left(\tau_{\varepsilon}\right), v_{\varepsilon}^{\prime}\left(\tau_{\varepsilon}\right)\right)=-\varepsilon\left(z_{\varepsilon}\left(\tau_{\varepsilon}\right), z_{\varepsilon}^{\prime}\left(\tau_{\varepsilon}\right)\right)
$$

so,

$$
\frac{T(\varepsilon(a, b))}{\varepsilon}=-\left(z_{\varepsilon}\left(\tau_{\varepsilon}\right), z_{\varepsilon}^{\prime}\left(\tau_{\varepsilon}\right)\right)
$$

By continuous dependence, it is clear that $\left(u_{\varepsilon}, z_{\varepsilon}\right)$ converges to the solution $(w, z)$ of

$$
\left\{\begin{array}{l}
w^{\prime \prime}+\nu w+w^{3}=0, \\
z^{\prime \prime}+\gamma\left(\nu+w^{2}\right) z=0, \\
w(0)=0, \quad w^{\prime}(0)=\sqrt{2 E_{0}}, \quad z(0)=a, \quad z^{\prime}(0)=b,
\end{array}\right.
$$

in $C^{1}([0, T])$ for every $T>0$. On the other hand, $L(a, b)=-\left(z(\rho), z^{\prime}(\rho)\right)$, where $\rho$ is the first positive zero of $w$. Therefore, to establish (2.4), it suffices to show that $\tau_{\varepsilon} \rightarrow \rho$, as $\varepsilon \downarrow 0$. This, however, is obvious since $w^{\prime}(0) \neq 0, w^{\prime}(\rho) \neq 0, w>0$ on $(0, \rho)$, and $\left(u_{\varepsilon}, z_{\varepsilon}\right) \rightarrow(w, z)$ in $C^{1}([0, \rho+1])$. This concludes the proof.

The main result of this section is the following.

Theorem 2.2. Fix $\gamma \in((m+1)(2 m+1),(m+1)(2 m+3))$ for some nonnegative integer $m$. If $E_{0}$ is sufficiently large, then there exist a nontrivial $C^{1}$-parametrized curve $\mathcal{C}$ in $\mathcal{U}$ passing through $(0,0)$ that is invariant under the action of $T$ and a constant $\delta \in(0,1)$ such that $|T(P)| \leq \delta|P|$ for all $P \in \mathcal{C}$.

The proof of Theorem 2.2 relies on the following result, which will be proved in Sec. 3 .

Theorem 2.3. Assume that $\gamma \in((m+1)(2 m+1),(m+1)(2 m+3))$ for some nonnegative integer $m$. If $E_{0}$ is sufficiently large, then the eigenvalues of $L$ are of the form $\lambda$ and $\lambda^{-1}$ for some $\lambda \in \mathbf{R}$ such that $0<|\lambda|<1$.

Proof of Theorem 2.2 (assuming Theorem 2.3). Using coordinates on $\mathbf{R}^{2}$ in which $L$ is diagonal, we have $L(x, y)=\left(\lambda x, \lambda^{-1} y\right)$. Proposition 2.1 then implies that $T(x, y)=$ $\left(\lambda x, \lambda^{-1} y\right)+F(x, y)$, for all $(x, y)$ in a neighborhood of $(0,0)$, where $F$ is $C^{1}$ and $F(0,0)=$ $0, D F(0,0)=0$. The result now follows from Lemma 5.1, p. 234 of Hartman [5].

We are now in a position to prove Theorem 1.1.

Proof of Theorem 1.1. Let $P=\left(v_{0}, v_{0}^{\prime}\right) \in \mathcal{C}$, the curve constructed in Theorem 2.2. it follows that

$$
\left|T^{n}(P)\right| \leq \delta^{n}|P|
$$

for all integers $n \geq 1$. Let $u_{0}^{\prime}>0$ be defined by

$$
\frac{u_{0}^{\prime 2}}{2}+\frac{v_{0}^{\prime 2}}{2 \gamma}+\nu \frac{v_{0}^{2}}{2}+\frac{v_{0}^{4}}{4}=E_{0},
$$

and let $(u, v)$ be the solution of (1.4) with initial data $\left(u, v, u^{\prime}, v^{\prime}\right)(0)=$ $\left(0, v_{0}, u_{0}^{\prime}, v_{0}^{\prime}\right)$. We denote by $\left(\tau_{n}\right)_{n \geq 1}$ the sequence of positive zeroes of $u$. It follows that $T\left(v_{0}, v_{0}^{\prime}\right)=-\left(v\left(\tau_{1}\right), v^{\prime}\left(\tau_{1}\right)\right) \in \mathcal{C}$, and $u^{\prime}\left(\tau_{1}\right)<0$ is given by

$$
\frac{u^{\prime}\left(\tau_{1}\right)^{2}}{2}+\frac{v^{\prime}\left(\tau_{1}\right)^{2}}{2 \gamma}+\nu \frac{v\left(\tau_{1}\right)^{2}}{2}+\frac{v\left(\tau_{1}\right)^{4}}{4}=E_{()} .
$$


If $\tilde{u}(t)=-u\left(\tau_{1}+t\right)$ and $\tilde{v}(t)=-v\left(\tau_{1}+t\right)$, then $(\tilde{u}, \tilde{v})$ solves (1.4), and $\tau_{2}-\tau_{1}$ is the first positive zero of $\tilde{u}$. It follows easily that $T\left(-v\left(\tau_{1}\right),-v^{\prime}\left(\tau_{1}\right)\right)=\left(v\left(\tau_{2}\right), v^{\prime}\left(\tau_{2}\right)\right)$. An obvious iteration argument shows that

$$
T^{n}\left(v_{0}, v_{0}^{\prime}\right)=(-1)^{n}\left(v\left(\tau_{n}\right), v^{\prime}\left(\tau_{n}\right)\right)
$$

and that $u^{\prime}\left(\tau_{n}\right)$ satisfies both $(-1)^{n} u^{\prime}\left(\tau_{n}\right)>0$ and

$$
\frac{u^{\prime}\left(\tau_{n}\right)^{2}}{2}+\frac{v^{\prime}\left(\tau_{n}\right)^{2}}{2 \gamma}+\nu \frac{v\left(\tau_{n}\right)^{2}}{2}+\frac{v\left(\tau_{n}\right)^{4}}{4}=E_{0} .
$$

Furthermore, successive zeroes of $u$ differ by less than $\pi / \sqrt{\nu}$ (multiply the equation by $\sin (t / \sqrt{\nu})$ and integrate by parts), i.e.,

$$
\tau_{n+1}-\tau_{n} \leq \pi / \sqrt{\nu}
$$

By conservation of energy, $u^{2}+v^{2}$ is a bounded function of $t$; so it follows from (1.4) and (2.10) that there exists a constant $C$, independent of $n$, such that

$$
v(t)^{2}+v^{\prime}(t)^{2} \leq C\left(v\left(\tau_{n}\right)^{2}+v^{\prime}\left(\tau_{n}\right)^{2}\right)
$$

for all $t \in\left[\tau_{n}, \tau_{n+1}\right]$. Applying now (2.6), (2.8), and (2.11), we get

$$
v(t)^{2}+v^{\prime}(t)^{2} \leq C \delta^{n}
$$

for all $t \in\left[\tau_{n}, \tau_{n+1}\right]$. Let $\varepsilon>0$ be such that $e^{-\varepsilon \pi / \sqrt{\nu}}=\delta$. It follows from (2.10) that $\tau_{n} \leq n \pi / \sqrt{\nu}$; so, for $t \in\left[\tau_{n}, \tau_{n+1}\right]$ we have

$$
e^{-\varepsilon t} \geq e^{-\varepsilon \tau_{n+1}} \geq e^{-\varepsilon(n+1) \pi / \sqrt{\nu}}=e^{-\varepsilon \pi / \sqrt{\nu}} \delta^{n} .
$$

Therefore, it follows from (2.12) that there exists $C$ such that

$$
v(t)^{2}+v^{\prime}(t)^{2} \leq C e^{-\varepsilon t}
$$

for all $t \geq 0$. This, together with (2.9), implies that there exists a constant $C$ such that

$$
\left|(-1)^{n} u^{\prime}\left(\tau_{n}\right)-\sqrt{2 E_{0}}\right| \leq C e^{-\varepsilon \tau_{n}} .
$$

Let now $w$ be the solution of the equation

$$
w^{\prime \prime}+\nu w+w^{3}=0
$$

with the initial data $w(0)=0$ and $w^{\prime}(0)=\sqrt{2 E_{0}}$. Comparing $(2.15)$ with the first equation in (1.4), and using the estimates (2.13) and (2.14) one can prove that there exists $C$ such that

$$
\left\|(-1)^{n} u\left(\tau_{n}+\cdot\right)-w(\cdot)\right\|_{C^{1}([0,2 \pi])} \leq C e^{-\varepsilon \tau_{n}}
$$


for all $n \geq 1$. If we denote by $\rho$ the first positive zero of $w$, it follows from (2.16) that there exists $C$ such that $\left|\tau_{n+1}-\tau_{n}-\rho\right| \leq C e^{-\varepsilon \tau_{n}}$. Also, (2.16) implies that $\tau_{n+1}-\tau_{n} \geq \rho / 2$ for $n$ large enough; so $\tau_{n} \geq n c$ for some constant $c>0$ and for $n$ large. Therefore, since $\tau_{n}-n \rho=\sum_{j=0}^{n-1}\left(\tau_{j+1}-\tau_{j}-\rho\right)$, where $\tau_{0}=0$, it follows that

$$
\left|\tau_{n}-n \rho-\theta\right| \leq C e^{-\varepsilon \tau_{n}}
$$

where $\theta=\sum_{j=0}^{\infty}\left(\tau_{j+1}-\tau_{j}-\rho\right)$. Since $w$ is clearly $\rho$ anti-periodic, i.e., $w(n \rho+\cdot)=$ $(-1)^{n} w(\cdot)$, it follows from (2.17) and continuous dependence of the solutions of Eq. (2.15) on the initial values that

$$
\left\|w(\cdot)-(-1)^{n} w\left(\tau_{n}-\theta+\cdot\right)\right\|_{C^{1}([0.2 \pi])}=\left\|w(n \rho+\cdot)-w\left(\tau_{n}-\theta+\cdot\right)\right\|_{C^{1}([0,2 \pi])} \leq C e^{-\varepsilon \tau_{n}} .
$$

Therefore, by (2.16),

$$
\left\|u\left(\tau_{n}+\cdot\right)-w\left(\tau_{n}-\theta+\cdot\right)\right\|_{C^{1}([0.2 \pi])} \leq C e^{-\varepsilon \tau_{n}},
$$

for all $n \geq 1$. This implies that

$$
\|u(t+\cdot)-w(t-\theta+\cdot)\|_{C^{1}([0,2 \pi])} \leq C e^{-\varepsilon t},
$$

for all $t \geq 0$. This estimate, together with (2.13), implies that $(u, v)$ has the asymptotic properties specified in Theorem 1.1. The theorem therefore follows by setting

$$
\mathcal{M}=\bigcup_{(a, b) \in \mathcal{C}} \bigcup_{t \in \mathbf{R}}\left(u(t), v(t), u^{\prime}(t), v^{\prime}(t)\right),
$$

where $(u, v)$ is the solution of $(1.1)$ with initial data $\left(u, v, u^{\prime}, v^{\prime}\right)(0)=\left(0, a, u_{0}^{\prime}, b\right)$ and $u_{0}^{\prime}>0$ is determined by $(2.7)$.

3. Analysis of the linearized system. This section is devoted to the proof of Theorem 2.3. In fact, we will prove a more precise result, giving a complete description of the operator $B_{\gamma}$ defined as follows. We denote by $w$ the solution of

$$
w^{\prime \prime}+w^{3}=0,
$$

with the initial values $w(0)=0$ and $w^{\prime}(0)=1$. We denote by $\theta$ the first positive zero of $w$. $w$ is odd and symmetric about $\theta / 2$, i.e., $w(t)=w(\theta-t)$. Given $\gamma \geq 0$, we consider the equation

$$
z^{\prime \prime}+\gamma w^{2} z=0
$$

For $(a, b) \in \mathbf{R}^{2}$, let $z$ be the solution of (3.2) with the initial values $z(0)=a$ and $z^{\prime}(0)=b$. The mapping $B_{\gamma} \in \mathcal{L}\left(\mathbf{R}^{2}\right)$ is defined by

$$
B_{\gamma}(a, b)=-\left(z(\theta), z^{\prime}(\theta)\right)
$$

We define the four sequences $\left(\mu_{n}^{-}\right)_{n \geq 0},\left(\mu_{n}^{+}\right)_{n \geq 0},\left(\gamma_{n}^{-}\right)_{n \geq 0}$, and $\left(\gamma_{n}^{+}\right)_{n \geq 0}$ by

$$
\begin{array}{ll}
\mu_{n}^{-}=2 n(4 n+1), & \gamma_{n}^{+}=(2 n+1)(4 n+1), \\
\mu_{n}^{+}=(2 n+1)(4 n+3), & \gamma_{n}^{-}=2(n+1)(4 n+3) ;
\end{array}
$$

we observe that $\mu_{n}^{-}<\gamma_{n}^{+}<\mu_{n}^{+}<\gamma_{n}^{-}<\mu_{n+1}^{-}$for all $n \geq 0$. The main result of this section is the following. 
THEOREM 3.1. The mapping $B_{\gamma}$ has the following properties.

(i) If $\gamma \in\left(\gamma_{n}^{+}, \mu_{n}^{+}\right)$for some $n \geq 0$, then the eigenvalues of $B_{\gamma}$ are $\lambda$ and $1 / \lambda$ for some $\lambda \in(0,1)$.

(ii) If $\gamma \in\left(\gamma_{n}^{-}, \mu_{n+1}^{-}\right)$for some $n \geq 0$, then the eigenvalues of $B_{\gamma}$ are $-\lambda$ and $-1 / \lambda$ for some $\lambda \in(0,1)$.

(iii) If $\gamma \in\left(\mu_{n}^{-}, \gamma_{n}^{+}\right) \cup\left(\mu_{n}^{+}, \gamma_{n}^{-}\right)$for some $n \geq 0$, then the eigenvalues of $B_{\gamma}$ are $e^{i \omega}$ and $e^{-i \omega}$ for some $\omega \in(0, \pi)$.

(iv) If $\gamma=\mu_{n}^{ \pm}$for some $n \geq 0$, then $B_{\gamma}=\left(\begin{array}{cc} \pm 1 & \pm a \\ 0 & \pm 1\end{array}\right)$ for some $a>0$.

(v) If $\gamma=\gamma_{n}^{ \pm}$for some $n \geq 0$, then $B_{\gamma}=\left(\begin{array}{cc} \pm 1 & 0 \\ \pm a \pm 1 & \pm 1\end{array}\right)$ for some $a>0$.

Proof of Theorem 2.3 (assuming Theorem 3.1). Let $\gamma$ be as in the statement of Theorem 2.3. Given $\varepsilon>0$, let $w_{\varepsilon}$ be the solution of the equation

$$
w_{\varepsilon}^{\prime \prime}+\varepsilon w_{\varepsilon}+w_{\varepsilon}^{3}=0
$$

with the initial data $w_{\varepsilon}(0)=0$ and $w_{\varepsilon}^{\prime}(0)=1$, and let $\theta_{\varepsilon}$ be the first positive zero of $w_{\varepsilon}$. For $(a, b) \in \mathbf{R}^{2}$, let $z_{\S}$ be the solution of the equation

$$
z_{\varepsilon}^{\prime \prime}+\gamma\left(\varepsilon+w_{\varepsilon}^{2}\right) z_{\varepsilon}=0
$$

with the initial data $z_{\varepsilon}(0)=a$ and $z_{\boldsymbol{g}}^{\prime}(0)=b$. We define the operator $B_{\gamma, \varepsilon} \in \mathcal{L}\left(\mathbf{R}^{2}\right)$ by $B_{\gamma, \varepsilon}(a, b)=-\left(z_{\varepsilon}\left(\theta_{\varepsilon}\right), z_{\varepsilon}^{\prime}\left(\theta_{\varepsilon}\right)\right)$. One verifies easily that $B_{\gamma, \varepsilon} \rightarrow B_{\gamma}$ as $\varepsilon \downarrow 0$. Therefore, it follows from Theorem 3.1 that there exists $\varepsilon_{0}>0$ such that for $\varepsilon \leq \varepsilon_{0}, B_{\gamma, \varepsilon}$ has a real eigenvalue $\lambda_{\gamma . \varepsilon}$ with $\left|\lambda_{\gamma, \varepsilon}\right| \in(0,1)$; so, there exists $(a, b) \in \mathbf{R}^{2}$ such that $\left(z_{\varepsilon}\left(\theta_{\varepsilon}\right), z_{\xi}^{\prime}\left(\theta_{\varepsilon}\right)\right)=$ $-\lambda_{\gamma, \varepsilon}(a, b)$. Let now

$$
w(t)=\sqrt{\frac{\nu}{\varepsilon}} w_{\varepsilon}\left(t \sqrt{\frac{\nu}{\varepsilon}}\right), \quad z(t)=z_{\varepsilon}\left(t \sqrt{\frac{\nu}{\varepsilon}}\right) .
$$

It follows that

$$
\left\{\begin{array}{l}
w^{\prime \prime}+\nu w+w^{3}=0, \\
z^{\prime \prime}+\gamma\left(\nu+w^{2}\right) z=0 .
\end{array}\right.
$$

Furthermore, $w(0)=0$ and $w^{\prime}(0)=\nu / \varepsilon$, and if we denote by $\rho$ the first positive zero of $w$, then $\rho=\sqrt{\varepsilon / \nu} \theta_{\varepsilon}$. A direct calculation shows that $\left(z(\rho), z^{\prime}(\rho)\right)=-\lambda_{\gamma, \varepsilon}\left(z(0), z^{\prime}(0)\right)$. Therefore, if $\sqrt{2 E_{0}} \geq \nu / \varepsilon_{0}$, then $L$ has the eigenvalue $\lambda_{\gamma . \varepsilon}$ with $\varepsilon=\left(2 E_{0}\right)^{-1 / 2} \nu$. Since $\tilde{z}$ defined by $\tilde{z}(t)=z(\rho-t)$ solves the same equation as does $z$, it follows that $1 / \lambda_{\gamma . \varepsilon}$ is the second eigenvalue of $L$. This completes the proof of the theorem.

The rest of this section is devoted to the proof of Theorem 3.1, which is long and requires several lemmas. We introduce four sequences of functions $\left(v_{n}^{ \pm}\right)_{n \geq 0}$ and $\left(u_{n}^{ \pm}\right)_{n \geq 0}$ as follows. For $n \geq 0$, we set

$$
\begin{array}{ll}
v_{n}^{--}(t)=P_{n}(x(t)), & u_{n}^{+}(t)=w(t) Q_{n}(x(t)), \\
v_{n}^{+}(t)=w^{\prime}(t) R_{n}(x(t)), & u_{n}^{-}(t)=w(t) w^{\prime}(t) S_{n}(x(t)) ;
\end{array}
$$

where

$$
x(t)=w(t)^{4}-1
$$


and $P_{n}, Q_{n}, R_{n}$, and $S_{n}$ are the Jacobi polynomials (see [9, $§ 2.2$ and $\left.\S 2.4\right]$ )

$$
\begin{array}{ll}
P_{n}(x)=a_{n} P_{n}^{\left(-\frac{1}{2},-\frac{1}{4}\right)}(x), & Q_{n}(x)=b_{n} P_{n}^{\left(-\frac{1}{2}, \frac{1}{4}\right)}(x), \\
R_{n}(x)=c_{n} P_{n}^{\left(\frac{1}{2},-\frac{1}{4}\right)}(x), & S_{n}(x)=d_{n} P_{n}^{\left(\frac{1}{2}, \frac{1}{4}\right)}(x),
\end{array}
$$

and where the constants $a_{n}, b_{n}, c_{n}$, and $d_{n}$ are chosen so that

$$
P_{n}(-1)=Q_{n}(-1)=R_{n}(-1)=S_{n}(-1)=1 .
$$

The introduction of these sequences of functions is justified by the following result.

LEMMA 3.2. Let the functions $\left(v_{n}^{ \pm}\right)_{n \geq 0}$ and $\left(u_{n}^{ \pm}\right)_{n \geq 0}$ be defined by (3.4) and the numbers $\left(\mu_{n}^{ \pm}\right)_{n \geq 0}$ and $\left(\gamma_{n}^{ \pm}\right)_{n \geq 0}$ be defined by $(3.3)$.

(i) $z=v_{n}^{ \pm}$is a solution of the equation $z^{\prime \prime}+\mu_{n}^{ \pm} w^{2} z=0$.

(ii) For every $n \geq 0, v_{n}^{ \pm}(0)=\mp v_{n}^{ \pm}(\theta)=1$ and $\left(v_{n}^{ \pm}\right)^{\prime}(0)=\left(v_{n}^{ \pm}\right)^{\prime}(\theta)=0$. Moreover, $v_{n}^{-}$ has exactly $2 n$ zeroes on $(0, \theta)$ and $v_{n}^{+}$has exactly $2 n+1$ zeroes on $(0, \theta)$.

(iii) $z=u_{n}^{ \pm}$is a solution of the equation $z^{\prime \prime}+\gamma_{n}^{ \pm} w^{2} z=0$.

(iv) For every $n \geq 0, u_{n}^{ \pm}(0)=u_{n}^{ \pm}(\theta)=0$ and $\left(u_{n}^{ \pm}\right)^{\prime}(0)=\mp\left(u_{n}^{ \pm}\right)^{\prime}(\theta)=1$. Moreover, $u_{n}^{+}$ has exactly $2 n$ zeroes on $(0, \theta)$ and $u_{n}^{-}$has exactly $2 n+1$ zeroes on $(0, \theta)$.

Proof. Properties (i) and (iii) follow from a direct calculation, since the Jacobi polynomial $y=P_{n}^{(a . b)}(x)$ satisfies the following ODE (see [9, Theorem 4.2.1, p. 60]):

$$
\left(1-x^{2}\right) y^{\prime \prime}+[b-a-(a+b+2) x] y^{\prime}+n(n+a+b+1) y=0 .
$$

Since $w$ is symmetric about $\theta / 2$ and since $w(\theta / 2)^{4}=2$, it follows that each real number in $(-1,1)$ is achieved exactly twice by the function $w(t)^{4}-1$ in the interval $(0, \theta)$. Therefore, since the Jacobi polynomial $P_{n}^{(a . b)}$ has exactly $n$ zeroes which are located in $(-1,1)$ (see $\left[9\right.$, Theorem 3.3 .1 , p. 44]), it follows that the functions $P_{n}\left(w^{4}-1\right), Q_{n}\left(w^{4}-1\right)$, $R_{n}\left(w^{4}-1\right)$, and $S_{n}\left(w^{4}-1\right)$ have exactly $2 n$ zeroes in $(0, \theta)$. Properties (ii) and (iv) follow immediately.

LEMMA 3.3. The mapping $B_{\gamma}$ has the following properties:

(i) $\operatorname{det} B_{\gamma}=1$.

(ii) If $\lambda$ is an eigenvalue of $B_{\gamma}$ corresponding to the eigenvector $(a, b)$, then $(a,-b)$ is also an eigenvector, corresponding to the eigenvalue $\lambda^{-1}$.

(iii) If $B_{\gamma}$ has the eigenvalue $\lambda= \pm 1$, then at least one of the vectors $(0,1)$ and $(1,0)$ is an eigenvector corresponding to the eigenvalue $\lambda$.

Proof. Let $z_{0}$ and $z_{1}$ be the solutions of $(3.2)$ with the initial data $(a, b)=(0,1)$ and $(a, b)=(1,0)$, respectively, and set $(\alpha, \beta)=-\left(z_{0}(\theta), z_{0}^{\prime}(\theta)\right)$ and $(\sigma, \delta)=-\left(z_{1}(\theta), z_{1}^{\prime}(\theta)\right)$. In other words, $(\alpha, \beta)=B_{\gamma}(0,1)$ and $(\sigma, \delta)=B_{\gamma}(1,0)$. It follows that

$$
B_{\gamma}=\left(\begin{array}{cc}
\sigma & \alpha \\
\delta & \beta
\end{array}\right) \text {. }
$$

Note that if $z$ solves (3.2), then $z(\theta-t)$ also solves (3.2). Therefore, $B_{\gamma}(z(\theta)$, $\left.-z^{\prime}(\theta)\right)=(-a, b)$. Choosing successively $(a, b)=(0,1)$ and $(a, b)=(1,0)$, we obtain

$$
\left\{\begin{array}{l}
\beta^{2}=\sigma^{2}=1+\alpha \delta \\
\alpha(\beta-\sigma)=\delta(\beta-\sigma)=0 .
\end{array}\right.
$$


It follows easily that either

$$
B_{\gamma}=\left(\begin{array}{cc} 
\pm 1 & 0 \\
0 & \mp 1
\end{array}\right)
$$

or else $\operatorname{det} B_{\gamma}=1$. We first prove (i) by contradiction, and so we assume that $\operatorname{det} B_{\gamma} \neq 1$. It follows from what precedes that (3.5) holds. Suppose first that

$$
B_{\gamma}=\left(\begin{array}{cc}
1 & 0 \\
0 & -1
\end{array}\right)
$$

It follows that $z_{0}(\theta)=0, z_{0}^{\prime}(\theta)=1, z_{1}(\theta)=-1$, and $z_{1}^{\prime}(\theta)=0$. This implies that $z_{0}(\theta-t)=-z_{0}(t)$ and that $z_{1}(\theta-t)=-z_{1}(t)$; so, $z_{0}(\theta / 2)=z_{1}(\theta / 2)=0$. Since $z_{0}$ and $z_{1}$ solve the same linear equation (i.e., (3.2)), it follows that $z_{0}$ and $z_{1}$ are linearly dependent, which is absurd. By an analogous argument, one excludes

$$
B_{\gamma}=\left(\begin{array}{cc}
-1 & 0 \\
0 & 1
\end{array}\right) ;
$$

so (i) is verified. Next, assume $B_{\gamma}(a, b)=\lambda(a, b)$. It follows from (i) that $\lambda \neq 0$. Let $z$ be the solution of $(3.2)$ with the initial data $\left(z(0), z^{\prime}(0)\right)=(a, b)$, and set $u(t)=z(\theta-t)$. Since $u(0)=z(\theta)=-\lambda a, u^{\prime}(0)=-z^{\prime}(\theta)=\lambda b, u(\theta)=z(0)=a$, and $u^{\prime}(\theta)=-z^{\prime}(0)=$ $-b$, it follows that $B_{\gamma}(-\lambda a, \lambda b)=(-a, b)$, i.e., $B_{\gamma}(a,-b)=\lambda^{-1}(a,-b)$. This proves property (ii). Assume now that $\lambda= \pm 1$ is an eigenvalue of $B_{\gamma}$ and let $(a, b)$ be an eigenvector corresponding to the eigenvalue $\lambda$. It follows from properties (i) and (ii) that (since $\left.\lambda=\lambda^{-1}\right) B_{\gamma}(a,-b)=\lambda(a,-b)$. If $(a, b)$ and $(a,-b)$ are linearly independent, then $B_{\gamma}=\lambda I$ and the conclusion of (iii) follows. If $(a, b)$ and $(a,-b)$ are linearly dependent, then either $a=0$ or else $b=0$, and the conclusion also follows.

We now study nodal properties of the solutions of Eq. (3.2). We begin with the following result.

LEMmA 3.4. Let $\gamma, \tilde{\gamma} \geq 0$ and let $u, \tilde{u} \not \equiv 0$ be such that $u^{\prime \prime}+\gamma w^{2} u=0$ and $\tilde{u}^{\prime \prime}+\tilde{\gamma} w^{2} \tilde{u}=0$.

(i) If $\gamma>\tilde{\gamma}$ and if $\sigma_{1}<\sigma_{2}$ are two consecutive zeroes of $\tilde{u}$, then $u$ has at least one zero on $\left(\sigma_{1}, \sigma_{2}\right)$.

(ii) If $\gamma=\tilde{\gamma}$ and if $\sigma_{1}<\sigma_{2}$ are two consecutive zeroes of $\tilde{u}$, then either $u$ and $\tilde{u}$ are linearly dependent, or else $u$ has exactly one zero on $\left(\sigma_{1}, \sigma_{2}\right)$.

(iii) If $\gamma>\tilde{\gamma}$ and if $\sigma_{1}<\sigma_{2}$ are such that $\tilde{u}^{\prime}\left(\sigma_{1}\right)=0, \tilde{u}\left(\sigma_{2}\right)=0$, and $\tilde{u} \neq 0$ on $\left(\sigma_{1}, \sigma_{2}\right)$, and if $u\left(\sigma_{1}\right)>0$ and $u^{\prime}\left(\sigma_{1}\right) \leq 0$ then $u$ has at least one zero on $\left(\sigma_{1}, \sigma_{2}\right)$.

Proof. (i) After possibly changing $\tilde{u}$ to $-\tilde{u}$, we may assume $\tilde{u}>0$ on $\left(\sigma_{1}, \sigma_{2}\right)$. We argue by contradiction and we assume (after possibly changing $u$ to $-u$ ) that $u>0$ on $\left(\sigma_{1}, \sigma_{2}\right)$. We have

$$
\left(u \tilde{u}^{\prime}-u^{\prime} \tilde{u}\right)^{\prime}=(\gamma-\tilde{\gamma}) w^{2} u \tilde{u}>0,
$$

almost everywhere on $\left(\sigma_{1}, \sigma_{2}\right)$ ("almost everywhere" due to the possible zeroes of $w$ on $\left.\left(\sigma_{1}, \sigma_{2}\right)\right)$. After integration over $\left(\sigma_{1}, \sigma_{2}\right)$, it follows that

$$
u\left(\sigma_{2}\right) \tilde{u}^{\prime}\left(\sigma_{2}\right)-u\left(\sigma_{1}\right) \tilde{u}^{\prime}\left(\sigma_{1}\right)>0,
$$

which is absurd since $\tilde{u}^{\prime}\left(\sigma_{1}\right)>0, \tilde{u}^{\prime}\left(\sigma_{2}\right)<0, u\left(\sigma_{1}\right) \geq 0$, and $u\left(\sigma_{2}\right) \geq 0$. This proves (i). 
(ii) If $u$ and $\tilde{u}$ are not linearly dependent, then in particular $u\left(\sigma_{1}\right) \neq 0$ and $u\left(\sigma_{2}\right) \neq 0$. After possibly changing $\tilde{u}$ to $-\tilde{u}$, we may assume $\tilde{u}>0$ on $\left(\sigma_{1}, \sigma_{2}\right)$. We argue by contradiction and we assume (after possibly changing $u$ to $-u$ ) that $u>0$ on $\left[\sigma_{1}, \sigma_{2}\right]$. Arguing as in the proof of (i), we obtain

$$
u\left(\sigma_{2}\right) \tilde{u}^{\prime}\left(\sigma_{2}\right)-u\left(\sigma_{1}\right) \tilde{u}^{\prime}\left(\sigma_{1}\right)=0,
$$

which is absurd since $\tilde{u}^{\prime}\left(\sigma_{1}\right)>0, \tilde{u}^{\prime}\left(\sigma_{2}\right)<0, u\left(\sigma_{1}\right)>0$, and $u\left(\sigma_{2}\right)>0$. Therefore, $u$ has a zero on $\left(\sigma_{1}, \sigma_{2}\right)$. If $u$ had two zeroes on $\left(\sigma_{1}, \sigma_{2}\right)$ then, by exchanging the roles of $u$ and $\tilde{u}$, we would obtain that $\tilde{u}$ would have a zero on $\left(\sigma_{1}, \sigma_{2}\right)$, which is absurd. This proves (ii).

(iii) We may assume that $u\left(\sigma_{1}\right)=\tilde{u}\left(\sigma_{1}\right)=1$ (since if $\tilde{u}\left(\sigma_{1}\right)=0$, then $\tilde{u} \equiv 0$ ). We argue by contradiction and we assume that $u>0$ on $\left(\sigma_{1}, \sigma_{2}\right)$. Arguing as in the proof of (i), we find

$$
u\left(\sigma_{2}\right) \tilde{u}^{\prime}\left(\sigma_{2}\right)+u^{\prime}\left(\sigma_{1}\right) \tilde{u}\left(\sigma_{1}\right)>0,
$$

which is absurd. This completes the proof.

Corollary 3.5. Let $\gamma, \tilde{\gamma} \geq 0$ and let $u, \tilde{u} \not \equiv 0$ be such that $u^{\prime \prime}+\gamma w^{2} u=0$ and $\tilde{u}^{\prime \prime}+\tilde{\gamma} w^{2} \tilde{u}=0$. Let $a<b$ and assume that $u$ has exactly $n$ zeroes on $(a, b)$ and that $\tilde{u}$ has exactly $\tilde{n}$ zeroes on $(a, b)$. If either $u(a)=u(b)=\tilde{u}(a)=\tilde{u}(b)=0$ or $u^{\prime}(a)=u^{\prime}(b)=\tilde{u}^{\prime}(a)=\tilde{u}^{\prime}(b)=0$, then the following properties hold.

(i) If $n>\tilde{n}$, then $\gamma>\tilde{\gamma}$.

(ii) If $n=\tilde{n}$, then $\gamma=\tilde{\gamma}$ and $u$ and $\tilde{u}$ are linearly dependent.

(iii) If $\gamma>\tilde{\gamma}$, then $n>\tilde{n}$.

(iv) If $\gamma=\tilde{\gamma}$, then $n=\tilde{n}$ and $u$ and $\tilde{u}$ are linearly dependent.

Proof. Assume first that $u(a)=u(b)=\tilde{u}(a)=\tilde{u}(b)=0$. Let $a=\sigma_{1}<\cdots<\sigma_{\tilde{n}+2}=b$ be the sequence of zeroes of $\tilde{u}$ on $[a, b]$. If $\gamma>\tilde{\gamma}$, then by applying Lemma 3.4(i) on each interval of the form $\left(\sigma_{j}, \sigma_{j+1}\right)$, we obtain that $u$ has at least $\tilde{n}+1$ zeroes on $(a, b)$. Hence (iii). If $\gamma=\tilde{\gamma}$, then $u$ and $\tilde{u}$ solve the same equation. Since this equation is linear and since $u(a)=\tilde{u}(a)=0$, it follows that $u$ and $\tilde{u}$ are linearly dependent, and in particular that $\tilde{n}=n$. Hence (iv). (i) and (ii) follow immediately. Assume now that $u^{\prime}(a)=u^{\prime}(b)=\tilde{u}^{\prime}(a)=\tilde{u}^{\prime}(b)=0$. Since $\tilde{u} \not \equiv 0$, we have $\tilde{u}(a) \neq 0$. Therefore, without loss of generality, we may assume $\tilde{u}(a)=1$. We first consider the case $\tilde{n} \geq 1$. Let $\left(\sigma_{i}\right)_{1 \leq i \leq n}$ be the sequence of zeroes of $\tilde{u}$ on $(a, b)$. If $\gamma>\tilde{\gamma}$, then by applying Lemma 3.4(i) on each interval of the form $\left(\sigma_{j}, \sigma_{j+1}\right)$ (if $\tilde{n} \geq 2$ ) and Lemma 3.4(iii) on the intervals $\left(a, \sigma_{1}\right)$ and $\left(\sigma_{\tilde{n}}, b\right)$, we obtain that $u$ has at least $\tilde{n}+1$ zeroes on $(a, b)$. Hence (iii). In the case $\tilde{n}=0$, we have $\tilde{\gamma}=0$. Indeed, otherwise we have $\tilde{u}>0$ on $(a, b)$, which implies that $\tilde{u}$ is strictly concave. This is absurd since $\tilde{u}^{\prime}(a)=\tilde{u}^{\prime}(b)=0$. If $n=0$, then the same argument shows that $\gamma=0$, and (iii) follows in this case also. If $\gamma=\tilde{\gamma}$, then $u$ and $\tilde{u}$ solve the same equation. Since this equation is linear and since $u^{\prime}(0)=\tilde{u}^{\prime}(0)=0$, it follows that $u$ and $\tilde{u}$ are linearly dependent, and in particular that $\tilde{n}=n$. Hence (iv). (i) and (ii) follow immediately.

Before stating the next result, we introduce some notation. Given $\gamma \geq 0$, we denote by $z_{\gamma}$ the solution of (3.2) such that

$$
z_{\gamma}(0)=0, \quad z_{\gamma}^{\prime}(0)=1
$$


and we denote by $\tilde{z}_{\gamma}$ the solution of (3.2) such that

$$
\tilde{z}_{\gamma}(0)=1, \quad \tilde{z}_{\gamma}^{\prime}(0)=0
$$

With this notation, we have the following result.

Lemma 3.6. For every $n \geq 0$, there exist $\sigma_{n}^{-} \in\left(\mu_{n}^{--}, \gamma_{n}^{+}\right)$and $\sigma_{n}^{+} \in\left(\mu_{n}^{+}, \gamma_{n}^{-}\right)$such that

(i) if $\gamma \in\left(\mu_{n}^{-}, \sigma_{n}^{-}\right)$, then $z_{\gamma}(\theta)>0, z_{\gamma}^{\prime}(\theta)>0, \tilde{z}_{\gamma}(\theta)>0$, and $\tilde{z}_{\gamma}^{\prime}(\theta)<0$;

(ii) if $\gamma \in\left(\sigma_{n}^{-}, \gamma_{n}^{+}\right)$, then $z_{\gamma}(\theta)>0, z_{\gamma}^{\prime}(\theta)<0, \tilde{z}_{\gamma}(\theta)<0$, and $\tilde{z}_{\gamma}^{\prime}(\theta)<0$;

(iii) if $\gamma \in\left(\gamma_{n}^{+}, \mu_{n}^{+}\right)$, then $z_{\gamma}(\theta)<0, z_{\gamma}^{\prime}(\theta)<0, \tilde{z}_{\gamma}(\theta)<0$, and $\tilde{z}_{\gamma}^{\prime}(\theta)<0$;

(iv) if $\gamma \in\left(\mu_{n}^{+}, \sigma_{n}^{+}\right)$, then $z_{\gamma}(\theta)<0, z_{\gamma_{i}^{\prime}}^{\prime}(\theta)<0, \tilde{z}_{\gamma}(\theta)<0$, and $\tilde{z}_{\gamma}^{\prime}(\theta)>0$;

(v) if $\gamma \in\left(\sigma_{n}^{+}, \gamma_{n}^{-}\right)$, then $z_{\gamma}(\theta)<0, z_{\gamma}^{\prime}(\theta)>0, \tilde{z}_{\gamma}(\theta)>0$, and $\tilde{z}_{\gamma}^{\prime}(\theta)>0$;

(vi) if $\gamma \in\left(\gamma_{n}^{-}, \mu_{n+1}^{-}\right)$, then $z_{\gamma}(\theta)>0, z_{\gamma}^{\prime}(\theta)>0, \tilde{z}_{\gamma}(\theta)>0$, and $\tilde{z}_{\gamma}^{\prime}(\theta)>0$.

Proof. We consider the functions $f, \tilde{f}, g, \tilde{g} \in C^{\infty}([0, \infty), \mathbf{R})$ defined by

$$
\begin{aligned}
f(\gamma)=z_{\gamma}(\theta), & g(\gamma)=z_{\gamma}^{\prime}(\theta), \\
\tilde{f}(\gamma)=\tilde{z}_{\gamma}(\theta), & \tilde{g}(\gamma)=\tilde{z}_{\gamma}^{\prime}(\theta) .
\end{aligned}
$$

It follows from Lemma 3.2 that

$$
\begin{aligned}
& f\left(\gamma_{n}^{ \pm}\right)=\tilde{g}\left(\mu_{n}^{ \pm}\right)=0, \\
& g\left(\gamma_{n}^{ \pm}\right)=\tilde{f}\left(\mu_{n}^{ \pm}\right)=\mp 1,
\end{aligned}
$$

for all $n \geq 0$. We claim that the $\gamma_{n}^{ \pm}$are the only zeroes of $f$, and that the $\mu_{n}^{ \pm}$are the only zeroes of $\tilde{g}$. Indeed, if $\gamma$ is a zero of $f$, then $z_{\gamma}(0)=z_{\gamma}(\theta)=0$, and it follows from Corollary 3.5 that $\gamma$ must be equal to one of the $\gamma_{n}^{ \pm}$'s. Similarly, if $\gamma$ is a zero of $\tilde{g}$, then $\tilde{z}_{\gamma}^{\prime}(0)=\tilde{z}_{\gamma}^{\prime}(\theta)=0$, and it follows from Corollary 3.5 that $\gamma$ must be equal to one of the $\mu_{n}^{ \pm}$'s. Next, set

$$
u_{\gamma}=\frac{\partial z_{\gamma}}{\partial \gamma}, \quad \tilde{u}_{\gamma}=\frac{\partial \tilde{z}_{\gamma}}{\partial \gamma}
$$

It follows that $u_{\gamma}$ solves the equation $u_{\gamma}^{\prime \prime}+\gamma w^{2} u_{\gamma}+w^{2} z_{\gamma}=0$ with the initial data $u_{\gamma}(0)=u_{\gamma}^{\prime}(0)=0$ and that $\tilde{u}_{\gamma}$ solves the equation $\tilde{u}_{\gamma}^{\prime \prime}+\gamma w^{2} \tilde{u}_{\gamma}+w^{2} \tilde{z}_{\gamma}=0$ with the initial data $\tilde{u}_{\gamma}(0)=\tilde{u}_{\gamma}^{\prime}(0)=0$. Since $\left(u_{\gamma} z_{\gamma}^{\prime}-u_{\gamma}^{\prime} z_{\gamma}\right)^{\prime}=w^{2} z_{\gamma}^{2}$ and $\left(\tilde{u}_{\gamma} \tilde{z}_{\gamma}^{\prime}-\tilde{u}_{\gamma}^{\prime} \tilde{z}_{\gamma}\right)^{\prime}=w^{2} \tilde{z}_{\gamma}^{2}$, it follows that

$$
\begin{aligned}
& u_{\gamma}(\theta) z_{\gamma}^{\prime}(\theta)-u_{\gamma}^{\prime}(\theta) z_{\gamma}(\theta)=\int_{0}^{\theta} w^{2} z_{\gamma}^{2}>0, \\
& \tilde{u}_{\gamma}(\theta) \tilde{z}_{\gamma^{\prime}}^{\prime}(\theta)-\tilde{u}_{\gamma}^{\prime}(\theta) \tilde{z}_{\gamma}(\theta)=\int_{0}^{\theta} w^{2} \tilde{z}_{\gamma}^{2}>0 .
\end{aligned}
$$

This means that

$$
\begin{gathered}
f^{\prime}(\gamma) g(\gamma)>f(\gamma) g^{\prime}(\gamma) \\
\tilde{f}^{\prime}(\gamma) \tilde{g}(\gamma)>\tilde{f}(\gamma) \tilde{g}^{\prime}(\gamma)
\end{gathered}
$$

In particular, $f$ and $f^{\prime}$ cannot vanish together, and similarly for $g, \tilde{f}$, and $\tilde{g}$. Therefore, the zeroes of $f, g, \tilde{f}$, and $\tilde{g}$ are isolated; and these functions always change sign at their 
zeroes. It also follows from (3.7) that the zeroes of $f$ and $g$ alternate, i.e., that between two consecutive zeroes of $f$ there is a zero of $g$ and between two consecutive zeroes of $g$ there is a zero of $f$. The same property obviously holds for the zeroes of $\tilde{f}$ and $\tilde{g}$. Therefore, $g$ must have exactly one zero on every interval of the form $\left(\gamma_{n}^{+}, \gamma_{n}^{-}\right)$and on every interval of the form $\left(\gamma_{n}^{-}, \gamma_{n+1}^{+}\right)$. Similarly, $\tilde{f}$ must have exactly one zero on every interval of the form $\left(\mu_{n}^{-}, \mu_{n}^{+}\right)$and on every interval of the form $\left(\mu_{n}^{+}, \mu_{n+1}^{-}\right)$. Furthermore, we claim that the zeroes of $g$ and $\tilde{f}$ coincide. Indeed, if $g(\gamma)=0$, then $z_{\gamma}^{\prime}(\theta)=0$. Setting

$$
v(t)=\frac{1}{z_{\gamma}(\theta)} z_{\gamma}(\theta-t)
$$

$\left(z_{\gamma}(\theta) \neq 0\right.$ since $\left.z_{\gamma} \not \equiv 0\right)$ it follows that $v$ solves Eq. $(3.2)$, that $v(0)=1, v^{\prime}(0)=0$, and $v(\theta)=0$; so, $\tilde{f}(\gamma)=0$. The converse statement is proved in the same way. We denote by $\sigma_{n}^{-}$the unique zero of $\tilde{f}$, and therefore of $g$, in $\left(\mu_{n}^{-}, \mu_{n}^{+}\right)$and by $\sigma_{n}^{+}$the unique zero of $\tilde{f}$, and therefore of $g$, in $\left(\mu_{n}^{+}, \mu_{n+1}^{-}\right)$. We claim that $\sigma_{0}^{-}<\gamma_{0}^{+}$. Indeed, since $\mu_{0}^{-}=0$, we know that $\sigma_{0}^{-}$is the first positive zero of $g$. On the other hand, since $z_{0}(t) \equiv t$, it follows that $g(0)=1$. Next, since $z_{1}=w$ and $\gamma_{0}^{+}=1$, it follows that $g\left(\gamma_{0}^{+}\right)=-1$; so, $g$ has a first zero in $\left(0, \gamma_{0}^{+}\right)$, and this first zero must be $\sigma_{0}^{-}$. This proves that $\sigma_{0}^{-}<\gamma_{0}^{+}$. Since $\sigma_{n}^{-}$is the unique zero of $g$ in $\left(\mu_{n}^{-}, \mu_{n}^{+}\right)$and $\sigma_{n}^{+}$the unique zero of $g$ in $\left(\mu_{n}^{+}, \mu_{n+1}^{-}\right)$, and since $g$ has a unique zero on every interval $\left(\gamma_{n}^{+}, \gamma_{n}^{-}\right)$and on every interval $\left(\gamma_{n}^{-}, \gamma_{n+1}^{+}\right)$, it follows easily that

$$
\mu_{n}^{-}<\sigma_{n}^{-}<\gamma_{n}^{+}<\mu_{n}^{+}<\sigma_{n}^{+}<\gamma_{n}^{-}<\mu_{n+1}^{-} .
$$

Finally, observe that $z_{0}(t) \equiv t$ and that $\tilde{z}_{0}(t) \equiv 1$, so that $f(0)=\theta>0, g(0)=1, \tilde{f}(0)=$ 1 , and $\tilde{g}(0)=0$. On the other hand, it follows from (3.7) that $\tilde{g}^{\prime}(0)<0$. Therefore, for $\gamma>0$ and small, we have $f(\gamma)>0, g(\gamma)>0, \tilde{f}(\gamma)>0$, and $\tilde{g}(\gamma)<0$. Since $0=\mu_{0}^{-}$, (i) through (vi) follow now immediately from the property that the zeroes of $f$ are the $\gamma_{n}^{ \pm}$, that the zeroes of $\tilde{g}$ are the $\gamma_{n}^{ \pm}$, and that the zeroes of $g$ and $\tilde{f}$ are the $\sigma_{n}^{ \pm}$.

Proof of Theorem 3.1. We use the notation of the proof of Lemma 3.6, and we observe that

$$
B_{\gamma}=-\left(\begin{array}{ll}
\tilde{f}(\gamma) & f(\gamma) \\
\tilde{g}(\gamma) & g(\gamma)
\end{array}\right)
$$

It follows that

$$
B_{\gamma^{ \pm}}=\left(\begin{array}{cc}
-\tilde{f}\left(\gamma_{n}^{ \pm}\right) & 0 \\
-\tilde{g}\left(\gamma_{n}^{ \pm}\right) & \pm 1
\end{array}\right) .
$$

In particular, \pm 1 is an eigenvalue of $B_{\gamma_{n}^{ \pm}}$. Since the other eigenvalue of $B_{\gamma_{n}^{ \pm}}$is also \pm 1 by Lemma 3.3, it follows that $-\tilde{f}\left(\gamma_{n}^{ \pm}\right)= \pm 1$. Finally, it follows easily from Lemma 3.6 that $\mp \tilde{g}\left(\gamma_{n}^{ \pm}\right)>0$; so

$$
B_{\gamma_{n}^{ \pm}}=\left(\begin{array}{cc} 
\pm 1 & 0 \\
\pm a_{n}^{ \pm} & \pm 1
\end{array}\right),
$$

where $a_{n}^{ \pm}>0$. This shows property (v), and one proves property (iv) in exactly the same way. Next, since $\operatorname{det} B_{\gamma}=1$, it follows that the eigenvalues of $B_{\gamma}$ are either $\{ \pm 1, \pm 1\}$, or $\left\{\lambda, \lambda^{-1}\right\}$ for some $\lambda \in(-1,1) \backslash\{0\}$, or else $\left(e^{i \omega}, e^{-i \omega}\right)$ for some $\omega \in(0, \pi)$. Furthermore, by Lemmas 3.3 and 3.6, the only values of $\gamma$ for which the eigenvalues of $B_{\gamma}$ are $\{ \pm 1, \pm 1\}$ are the $\gamma_{n}^{ \pm}$and the $\mu_{n}^{ \pm}$. Therefore, in the interval $\left(\mu_{n}^{-}, \gamma_{n}^{+}\right)$, the eigenvalues 
of $B_{\gamma}$ are either of the form $\left\{\lambda(\gamma), \lambda(\gamma)^{-1}\right\}$ for some $\lambda(\gamma) \in(-1,1) \backslash\{0\}$ or else of the form $\left\{e^{i \omega(\gamma)}, e^{-i \omega(\gamma)}\right\}$ for some $\omega \in(0, \pi)$. Since the eigenvalues depend continuously on $\gamma$ and since for $\mu_{n}^{-}$they are $\{-1,-1\}$ and for $\gamma_{n}^{+}$they are $\{1,1\}$, they must be of the form $\left\{e^{i \omega(\gamma)}, e^{-i \omega(\gamma)}\right\}$ for some $\omega \in(0, \pi)$. This proves the first statement of property (iii). The second statement is proved in exactly the same way. Let now $\gamma \in\left(\gamma_{n}^{+}, \mu_{n}^{+}\right)$. It follows from property (iii) of Lemma 3.6 that

$$
B_{\gamma}=\left(\begin{array}{ll}
a & c \\
b & d
\end{array}\right)
$$

for some $a, b, c, d>0$ such that $a d-b c=1$. This implies that the eigenvalues of $B$, are $\lambda$ and $\lambda^{-1}$ for some $\lambda \in(0,1)$. This proves property (i). The proof of property (ii) is quite similar.

4. Further results. In this section, we present two extensions of Theorem 1.1. First, we consider Eq. (1.1) with $a=0$, in which case we have an analogue of Theorem 1.1, without the energy condition. Next, we consider a nonlinearity more general than in Eq. (1.1), and for which we still can prove an analogue of Theorem 1.1. We begin with the case $a=0$, which corresponds to $\nu=0$ in Eq. (1.4), and for which the following improvement of Theorem 1.1 can be proved.

Theorem 4.1. Let $\nu=0$ in Eq. (1.4). Assume that

$$
\gamma \in((m+1)(2 m+1),(m+1)(2 m+3))
$$

for some nonnegative integer $m$. For every $E_{0}>0$, there exists a two-dimensional submanifold $\mathcal{M}$ of the (three-dimensional) manifold $\left\{E\left(u, v, u^{\prime}, v^{\prime}\right)=E_{0}\right\}$ with the following property. If $\left(u_{0}, v_{0}, u_{0}^{\prime}, v_{0}^{\prime}\right) \in \mathcal{M}$, and if $(u, v)$ is the solution of (1.4) (with $\nu=0$ ) with initial data $\left(u_{0}, v_{0}, u_{0}^{\prime}, v_{0}^{\prime}\right)$, then $v$ and $v^{\prime}$ converge exponentially to 0 as $t \rightarrow \infty$, and there exists a solution $w$ of the equation $w^{\prime \prime}+w^{3}=0$ with energy $\frac{w^{\prime 2}}{2}+\frac{u^{4}}{4}=E_{0}$ such that $u-w$ and $u^{\prime}-w^{\prime}$ converge exponentially to 0 as $t \rightarrow \infty$.

Proof. The proof is a variation of the proof of Theorem 1.1, and we only indicate the modifications. In this case, a scaling argument, similar to the one in the proof of Theorem 2.3, shows that

$$
L=J B_{\gamma} J^{-1}
$$

where

$$
J=\left(\begin{array}{cc}
1 & 0 \\
0 & \sqrt{w_{0}^{\prime}}
\end{array}\right),
$$

and $B_{\gamma}$ is defined in Sec. 3. In particular, the eigenvalues of $L$ are the eigenvalues of $B_{\gamma}$; so, they are given by Theorem 3.1. Therefore, in the case $\nu=0$, the conclusion of Theorem 2.3, hence the conclusion of Theorem 2.2 hold without the energy condition. The proof is now the same as the proof of Theorem 1.1 (see the end of Sec. 2), except that inequality $(2.10)$ is not anymore meaningful, and so one must show that there exists a constant $T$, independent of $n$, such that

$$
\tau_{n+1}-\tau_{n} \leq T
$$


This implies, instead of (2.16), the following inequality:

$$
\left\|(-1)^{n} u\left(\tau_{n}+\cdot\right)-w(\cdot)\right\|_{C^{1}([0.2 \eta])} \leq C e^{-\varepsilon \tau_{n}},
$$

and the rest of the proof is unchanged. Therefore, we need only prove estimate (4.1). First of all, one verifies quite easily that $u$ has infinitely many zeroes, i.e., that the $\tau_{n}$ 's are well defined. Next, by $(2.6)$ and $(2.8)$, it follows that $v\left(\tau_{n}\right)^{2}+v^{\prime}\left(\tau_{n}\right)^{2} \rightarrow 0$; so by $(2.9)$

$$
\left|u^{\prime}\left(\tau_{n}\right)\right| \geq \eta
$$

for all $n \geq 0$. Also, by conservation of energy, there exists $M<\infty$ such that

$$
u^{\prime}(t)^{2}+u(t)^{2}+v(t)^{2} \leq M^{2}
$$

for all $t \geq 0$. Now fix $n \geq 0$ and assume, for example, that $u^{\prime}\left(\tau_{n}\right) \geq 0$. It follows that $u$ is positive and concave on $\left(\tau_{n}, \tau_{n+1}\right)$ and thus has a unique maximum in $\left(\tau_{n}, \tau_{n+1}\right)$, achieved at $\sigma_{n} \in\left(\tau_{n}, \tau_{n+1}\right)$. Clearly, we only need to estimate $\sigma_{n}-\tau_{n}$ in terms of $M$ and $\eta$, since then an estimate for $\tau_{n+1}-\sigma_{n}$ will follow by changing $t$ to $\tau_{n+1}-t$. Let $s \in\left(\tau_{n}, \sigma_{n}\right)$ be such that

$$
M \geq u^{\prime}(s)=\frac{u^{\prime}\left(\tau_{n}\right)}{2} \geq \frac{\eta}{2}
$$

Since $u^{\prime} \geq \frac{\eta}{2}$ on $\left[\tau_{n}, s\right]$, we have

$$
u(t) \geq \frac{\eta}{2}\left(t-\tau_{n}\right)
$$

so,

$$
s-\tau_{n} \leq \frac{2 M}{\eta}
$$

In addition, since $u^{\prime \prime} \geq-M^{3}$, it follows that $u^{\prime}(t) \geq \eta-M^{3}\left(t-\tau_{n}\right)$. Choosing $t=s$, we obtain

$$
s-\tau_{n} \geq \frac{\eta}{2 M^{3}} .
$$

It follows from (4.4) and (4.6) that

$$
u(s) \geq \frac{\eta^{2}}{4 M^{3}}
$$

Next, since $u(s) \leq u(t) \leq M$ on $\left[s, \sigma_{n}\right]$, we have

$$
u^{\prime \prime}(t) \leq-u(s)^{3}
$$

on $\left[s, \sigma_{n}\right] ;$ so,

$$
0 \leq u^{\prime}(t) \leq M-(t-s) u(s)^{3},
$$

from which it follows, by applying (4.7), that

$$
\sigma_{n}-s \leq 64 \frac{M^{10}}{\eta^{6}} .
$$


The result now follows from the above inequality and (4.5).

Next, in order to extend Theorem 2.1 to include more general nonlinearities, we begin by generalizing Theorem 3.1. Let $\alpha>0$ and consider the solution $w$ of

$$
w^{\prime \prime}+|w|^{\alpha} w=0
$$

with the initial values $w(0)=0$ and $w^{\prime}(0)=1$. We denote by $\theta$ the first positive zero of $w$. $w$ is odd and symmetric about $\theta / 2$, i.e., $w(t)=w(\theta-t)$. Given $\gamma \geq 0$, we consider the equation

$$
z^{\prime \prime}+\gamma|w|^{\alpha} z=0
$$

For $(a, b) \in \mathbf{R}^{2}$, let $z$ be the solution of (4.9) with the initial values $z(0)=a$ and $z^{\prime}(0)=b$. We define the mapping $B_{\gamma} \in \mathcal{L}\left(\mathbf{R}^{2}\right)$ by

$$
B_{\gamma}(a, b)=-\left(z(\theta), z^{\prime}(\theta)\right)
$$

We define the four sequences $\left(\mu_{n}^{-}\right)_{n \geq 0},\left(\mu_{n}^{+}\right)_{n \geq 0},\left(\gamma_{n}^{-}\right)_{n \geq 0}$, and $\left(\gamma_{n}^{+}\right)_{n \geq 0}$ by

$$
\begin{array}{ll}
\mu_{n}^{-}=n((2(\alpha+2) n+\alpha)), & \gamma_{n}^{+}=(2 n+1)((\alpha+2) n+1), \\
\mu_{n}^{+}=(2 n+1)((\alpha+2) n+\alpha+1), & \gamma_{n}^{-}=(n+1)(2(\alpha+2) n+\alpha+4),
\end{array}
$$

and we observe that $\mu_{n}^{-}<\gamma_{n}^{+}<\mu_{n}^{+}<\gamma_{n}^{-}<\mu_{n+1}^{-}$for all $n \geq 0$. Note that if $\alpha=2$, then $B_{\gamma}$ and the various constants reduce to those defined in Sec. 3. Moreover, Theorem 3.1 is still valid in the present, more general context.

ThEOREM 4.2. Let $\alpha>0$. If $B_{\gamma}, \mu_{n}^{ \pm}$, and $\gamma_{n}^{ \pm}$are as just defined, then $B_{\gamma}$ has all the properties specified in Theorem 3.1.

Proof. The proof is quite similar to the proof of Theorem 3.1, except that the four sequences of functions $\left(v_{n}^{ \pm}\right)_{n \geq 0}$ and $\left(u_{n}^{ \pm}\right)_{n \geq 0}$ are defined by (3.4) where now

$$
x(t)=\frac{4}{\alpha+2} w(t)^{\alpha+2}-1,
$$

and $P_{n}, Q_{n}, R_{n}$, and $S_{n}$ are the Jacobi polynomials (see $[9, \S 2.2$ and $\S 2.4]$ )

$$
\begin{array}{ll}
P_{n}(x)=a_{n} P_{n}^{\left(-\frac{1}{2} \cdot-\frac{1}{1+\frac{1}{2}}\right)}(x), & Q_{n}(x)=b_{n} P_{n}^{\left(-\frac{1}{2} \cdot \frac{1}{n+2}\right)}(x), \\
R_{n}(x)=c_{n} P_{n}^{\left(\frac{1}{2} \cdot-\frac{1}{\left.x+\frac{1}{2}\right)}\right)}(x), & S_{n}(x)=d_{n} P_{n}^{\left(\frac{1}{2} \cdot \frac{1}{n+2}\right)}(x),
\end{array}
$$

and where the constants $a_{n}, b_{n}, c_{n}$, and $d_{n}$ are chosen so that

$$
P_{n}(-1)=Q_{n}(-1)=R_{n}(-1)=S_{n}(-1)=1 \text {. }
$$

With this notation, the proof of Theorem 4.1 follows the proof of Theorem 3.1 with obvious modifications. In particular, the conclusions of Lemma 3.2 are valid as stated in the present context, for all $\alpha>0$. 
REMARK. The functions $\left(v_{n}^{ \pm}\right)_{n \geq 0}$ and $\left(u_{n}^{ \pm}\right)_{n \geq 0}$ can be calculated directly by using the following recurrence formulas:

$$
\begin{array}{rlrl}
v_{0}^{-}(t) & \equiv 1, \quad u_{0}^{+}(t) \equiv w(t), & & \\
v_{n}^{+} & =\frac{\left(v_{n}^{-}\right)^{\prime} w}{n(\alpha+2)+\frac{\alpha}{2}}+v_{n}^{-} w^{\prime}, & v_{n+1}^{-}=\frac{\left(v_{n}^{+}\right)^{\prime} w}{n(\alpha+2)+\alpha+1}+v_{n}^{+} w^{\prime}, \\
u_{n}^{-} & =\frac{\left(u_{n}^{+}\right)^{\prime} w+(2 n+1)\left(\frac{\alpha}{2}+1\right) u_{n}^{+} w^{\prime}}{(2 n+1)\left(\frac{\alpha}{2}+1\right)+1}, & & u_{n+1}^{+}=\frac{\left(u_{n}^{-}\right)^{\prime} w+(n+1)(\alpha+2) u_{n}^{-} w^{\prime}}{(n+1)(\alpha+2)+1} .
\end{array}
$$

It is straightforward to check that these recurrence relations generate solutions to the appropriate equations with the appropriate initial data. By uniqueness, these functions are the same as those defined in the proof of Theorem 4.2.

Let now $f:[0, \infty) \rightarrow \mathbf{R}$ satisfy the following properties:

$$
\begin{aligned}
& f \in C([0, \infty)) \cap C^{1}((0, \infty)), \quad f(0)=0, \quad s f^{\prime}(s) \underset{s \downarrow 0}{\longrightarrow} 0, \\
& \inf _{s \geq 0} f(s)>-1, \quad f(s) \underset{s \rightarrow \infty}{\longrightarrow}+\infty, \quad \frac{f(t x)}{f(t)} \underset{t \rightarrow \infty}{\longrightarrow}|x|^{\alpha},
\end{aligned}
$$

where $\alpha$ is a positive number and the last limit is uniform for bounded values of $x$. Note that nonlinearities of the form $f(x)=b x^{\alpha}(\log (1+x))^{q}$, for $b>0, \alpha>0$, and $q \geq 0$ verify assumption (4.10). We consider the equation

$$
\left\{\begin{array}{l}
u_{t t}-a\left(1+f\left(\int_{0}^{\pi} u_{x}^{2}(t, x) d x\right)\right) u_{x x}=0, \\
u_{\mid \partial \Omega}=0,
\end{array}\right.
$$

in $\Omega=(0, \pi)$, where $a>0$. Expanding $u$ in a Fourier series, i.e., setting

$$
u_{j}(t)=\sqrt{\frac{2}{\pi}} \int_{0}^{\pi} u(t, x) \sin (j x) d x,
$$

we obtain for the $u_{j}$ 's the following system of ODE's:

$$
u_{j}^{\prime \prime}+a j^{2}\left(1+f\left(\sum_{i=1}^{\infty} i^{2} u_{i}^{2}\right)\right) u_{j}=0 .
$$

It follows that, given any integer $j \geq 1$, Eq. (4.11) has the particular (simple mode) solution $u(t, x)=\sqrt{\frac{2}{\pi}} \omega(t) \sin (j x)$ where $\omega$ solves the equation $\omega^{\prime \prime}+j^{2}\left(a+f\left(j^{2} \omega^{2}\right)\right) \omega=0$. We consider a two-mode solution of (4.11) (or (4.12)), i.e., a solution of the system

$$
\left\{\begin{array}{l}
u_{j}^{\prime \prime}+a j^{2}\left(1+f\left(j^{2} u_{j}^{2}+k^{2} u_{k}^{2}\right)\right) u_{j}=0, \\
u_{k}^{\prime \prime}+a k^{2}\left(1+f\left(j^{2} u_{j}^{2}+k^{2} u_{k}^{2}\right)\right) u_{k}=0,
\end{array}\right.
$$

where $j, k$ are integers such that $k>j \geq 1$. If $\left(u_{j}, u_{k}\right)$ solves system (4.13), then $u(t, x)=\sqrt{\frac{2}{\pi}}\left(u_{j}(t) \sin (j x)+u_{k}(t) \sin (k x)\right)$ solves Eq. (4.11). We set

$$
\gamma=\frac{k^{2}}{j^{2}}, \quad \sigma=j \sqrt{a},
$$


so that (4.13) is equivalent to

$$
\left\{\begin{array}{l}
u^{\prime \prime}+\left(1+f\left(u^{2}+v^{2}\right)\right) u=0 \\
v^{\prime \prime}+\gamma\left(1+f\left(u^{2}+v^{2}\right)\right) v=0
\end{array}\right.
$$

where $j u_{j}(t)=u(\sigma t)$ and $k u_{k}(t)=v(\sigma t)$. The system (4.14) is a Hamiltonian system, whose conserved energy, expressed in terms of $u, v, u^{\prime}, v^{\prime}$, is given by

$$
E\left(u, v, u^{\prime}, v^{\prime}\right)=\frac{1}{2}\left(u^{\prime 2}+\frac{v^{\prime 2}}{\gamma}+u^{2}+v^{2}+F\left(u^{2}+v^{2}\right)\right),
$$

where

$$
F(x)=\int_{0}^{x} f(s) d s
$$

We have the following analogue of Theorem 1.1.

THEOREM 4.3. Let $f$ verify (4.10), and assume that

$$
\gamma \in\left((m+1)\left(\frac{\alpha+2}{2} m+1\right),(m+1)\left(\frac{\alpha+2}{2} m+1+\alpha\right)\right),
$$

for some nonnegative integer $m$. There exists $C>0$ such that if $E_{0} \geq C$, then there exists a two-dimensional submanifold $\mathcal{M}$ of the (three-dimensional) manifold $\left\{E\left(u, v, u^{\prime}, v^{\prime}\right)=\right.$ $\left.E_{0}\right\}$ with the following property. If $\left(u_{0}, v_{0}, u_{0}^{\prime}, v_{0}^{\prime}\right) \in \mathcal{M}$, and if $(u, v)$ is the solution of (4.14) with initial data $\left(u_{0}, v_{0}, u_{0}^{\prime}, v_{0}^{\prime}\right)$, then $v$ and $v^{\prime}$ converge exponentially to 0 as $t \rightarrow \infty$, and there exists a solution $w$ of the equation $w^{\prime \prime}+w+f\left(w^{2}\right) w=0$ with energy $\frac{1}{2}\left(w^{\prime 2}+w^{2}+F\left(w^{2}\right)\right)=E_{0}$ such that $u-w$ and $u^{\prime}-w^{\prime}$ converge exponentially to 0 as $t \rightarrow \infty$.

Proof. The proof is an adaptation of the proof of Theorem 1.1 with the following modifications. The linearized system is

$$
\left\{\begin{array}{l}
w^{\prime \prime}+w+f\left(w^{2}\right) w=0 \\
z^{\prime \prime}+\gamma\left(1+f\left(w^{2}\right)\right) z=0
\end{array}\right.
$$

with the initial conditions $w(0)=0, w^{\prime}(0)=\sqrt{2 E_{0}}, z(0)=a$, and $z^{\prime}(0)=b$. Let $g:[0, \infty) \rightarrow \mathbf{R}$ be a positive, continuous function such that

$$
g(t)=\sqrt{f\left(t^{2}\right)}
$$

for $t$ large. It follows from (4.10) that such a function exists and that

$$
\frac{f\left(\lambda^{2} x^{2}\right)}{g(\lambda)^{2}}=\frac{f\left(\lambda^{2} x^{2}\right)}{f\left(\lambda^{2}\right)} \frac{f\left(\lambda^{2}\right)}{g(\lambda)^{2}} \underset{\lambda \rightarrow x}{\longrightarrow}|x|^{2 \alpha},
$$

uniformly for $x$ in a bounded set. For every $E_{0}>0$, set $\lambda=\sup \left\{t>0 ; \operatorname{tg}(t)=\sqrt{2 E_{0}}\right\}$. It follows from assumption (4.10) that $\lambda$ is well defined, that

$$
\lambda g(\lambda)=\sqrt{2 E_{0}}=w^{\prime}(0),
$$


and that

$$
\lambda \underset{E_{0} \rightarrow \infty}{\longrightarrow} \infty, \quad g(\lambda) \underset{E_{0} \rightarrow \infty}{\longrightarrow} \infty .
$$

Define $x$ and $y$ by $w(t)=\lambda x(g(\lambda) t)$ and $z(t)=y(g(\lambda) t)$. It follows that $(x, y)$ solves the system

$$
\left\{\begin{array}{c}
x^{\prime \prime}+\frac{1}{g(\lambda)^{2}} x+\frac{f\left(\lambda^{2} x^{2}\right)}{g(\lambda)^{2}} x=0, \\
y^{\prime \prime}+\frac{\gamma}{g(\lambda)^{2}} y+\gamma \frac{f\left(\lambda^{2} x^{2}\right)}{g(\lambda)^{2}} y=0,
\end{array}\right.
$$

with the initial conditions $x(0)=0, x^{\prime}(0)=1$ (by $\left.(4.3)\right), y(0)=a$, and $y^{\prime}(0)=b g(\lambda)^{-1}$. By applying Theorem 4.2 instead of Theorem 3.1, one obtains an analogue of Theorem 2.3. The rest of the proof is completely analogous to the proof of Theorem 1.1.

\section{REFERENCES}

[1] S. Bernstein, Sur une classe d'équations fonctionnelles aux dérivées partielles, Izv. Acad. Nauk. SSSR, Ser. Math. 4, 17-26 (1940)

[2] G. F. Carrier, On the vibration problem of an elastic string, Quart. Appl. Math. 3, 151-165 (1945)

[3] T. Cazenave and F. B. Weissler, Asymptotically periodic solutions for a class of nonlinear coupled oscillators, Portugaliæ Math. 52, 109-123 (1995)

[4] R. W. Dickey, Stability of periodic solutions of the nonlinear string equation, Quart. Appl. Math. 38, 253-259 (1980)

[5] P. Hartman, Ordinary Differential Equations, John Wiley and Sons, New York, 1964

[6] W. Magnus and S. Winkler, Hill's equation, Interscience Tracts in Pure and Applied Mathematics \# 20, Interscience Publishers, New York, 1966

[7] L. A. Medeiros and M. Milla Miranda, Solution for the equation of nonlinear vibration in Sobolev spaces of fractionary order, Mat. Aplic. Comp. 6, 257 276 (1987)

[8] R. Narashimha, Nonlinear vibrations of an elastic string, J. Sound Vib. 8, $134 \cdots 136$ (1968)

[9] G. Szegö, Orthogonal Polynomials, Amer. Math. Soc., Providence, RI, 1967 